Journal of the Egyptian Society of Parasitology, Vol.43, No.3, December 2013

J. Egypt. Soc. Parasitol., 43(3), 2013: 689 - 696

\title{
SIALIC ACID VALUE IN PLEURAL EFFUSION AS A DIAGNOSTIC MARKER OF MALIGNANCY \\ By

\author{
SHERIF ALSAYED ${ }^{1}$ AND SAMAR MARZOUK ${ }^{2}$
}

Department of chest ${ }^{1}$, Faculty of Medicine, El Fayoum, University, Al Fayoum, and Department Biochemistry ${ }^{2}$, Faculty of Medicine, Cairo University, Cairo, Egypt

\section{Abstract}

In differential diagnosis of pleural effusions, cytology is the most sensitive method. Since cytology findings are positive in half of such fluids, combined use of reliable tumor marker and cytology is a logical approach. Sialic acid and other tumor markers can be reliable substances associated with neoplasia.

The present study measured sialic acid levels in pleural effusion and serum samples of patients with malignant and nonmalignant diseases to discriminate each other, and to diagnose malignant effusion in a simple, cheap and reliable way. Sixty patients with pleural effusion were enrolled in the study and classified into two groups, group (I) 30 patients with malignant pleural effusion, and group (II) 30 patients with nonmalignant pleural effusion. Pleural fluid (PF) and serum (S) levels of sialic acid were measured prior to any therapy; and $\mathrm{PF} / \mathrm{S}$ sialic acid ratios were calculated.

Pleural fluid and serum levels of sialic acid were significantly higher in malignant group compared to nonmalignant one. $\mathrm{PF} / \mathrm{S}$ sialic acid ratio was higher in malignant group compared to nonmalignant group. In the malignant pleural effusion group, smokers showed a statistically significant higher pleural fluid and serum sialic acid levels as compared to nonsmokers. By using ROC curve, the cut off value of malignant pleural fluid sialic acid was $69.65 \mathrm{mg} / \mathrm{dL}$, sensitivity was $70 \%$, and specificity was $96 \%$.

Key words: Sialic acid, pleural effusion, lung neoplasms.

\section{Introduction}

Pleural effusion is an excessive accumulation of fluid in the pleural space, indicates an imbalance between pleural fluid formation and removal. Pleural effusions accompany a wide variety of disorders of the lung, pleura, and systemic disorders (Vinaya and Jyotsna, 2012).

The most common conditions that result in effusions are cardiac failure, pneumonia, and malignant neoplasm. Malignant disease involving pleura is the second leading cause of exudative pleural effusions after para-pneumonic effusions (Emmet and Paul, 2011).

It is important to elucidate the precise etiology to differentiate benign from malignant effusions. The initial diagnostic approach includes thoracocentesis and cytologic, histologic, and biochemical examinations (Light, 1995). 
However, the sensitivity of these noninvasive techniques is only $40 \%-70 \%$ (Marel et al, 1995). To improve upon these rates, a number of tumor markers in the pleural fluid have been intensively evaluated and reported to have diagnostic rule or value in malignant effusion (Villena et al, 1996).

One of these markers is sialic acid (SA). Sialic acid levels had been found to be elevated in neoplastic cells derived from lung, breast, stomach, colon, ovary, prostate and liver. Recently sialic acid had been reported to have a diagnostic value in malignancy (Nicola and Prasad, 2002).

Sialic acid is the generic term given to a family of acetylated derivatives of neuraminic acid (N-acetyl neuraminic acid; NANA), which occur mainly at terminal positions of glycoprotein and glycolipid oligosaccharide side chains. Several biological functions have been suggested for SA, such as stabilizing the conformation of glycoproteins and cellular membranes, assisting in cellcell recognition and interaction, contributing to membrane transport, providing binding sites for ligands for the membrane receptor functions, and affecting the function, stability and survival of glycoproteins in blood circulation (Selva and Kalaivani, 2011).

The present study aimed to measure Sialic acid levels in pleural effusion and serum samples of patients with malignant and nonmalignant diseases to discriminate each other, and to diagnose malignant effusion in a simple, cheap and reliable way.

\section{Subjects, Materials and Methods}

Sixty patients with pleural effusion were enrolled and classified into two groups as follows:

GI $(n=30)$ : patients with malignant pleural effusion (proved malignant by pleural biopsy), they were 11 females and 19 males, 25 with lung cancer, 3 with mesothelioma, 1 with esophageal neoplasia, and 1 with mediastinal neoplasia (age range 25 to 72 years).

GII $(n=30)$ : control patients with nonmalignant pleural effusion, they were 8 females and 22 males, 20 with tuberculosis, 5 with pneumonia, 3 with cardiac failure, 1 with liver cirrhosis, and 1 with chronic renal failure (age range 26 to 70 years). 16 patients from group (I) were smokers, while 19 patients from group (II) were smokers.

All patients were recruited from the NJCH Outpatients' Clinic of Chest Department, in the years 2012-2013. All participants gave their informed consent before participation in the study, and the Ethics Committee of $\mathrm{NJCH}$ Hospital approved the protocol of the study.

They were subjected to detailed clinical history, thorough clinical examination and routine investigation. None of the patients was receiving drugs known to affect the investigated parameter.

Pleural fluid and serum samples were collected from each patient prior to any therapy. The supernatant of pleural fluid samples were obtained by centrifugation at $300 \mathrm{rpm}$ for 15 minutes and stored at $-20^{\circ} \mathrm{C}$ until assayed. $10 \mathrm{ml}$ blood was collected from the antecubital vein, centrifuged at 3,000 rpm for 10 minutes at room temperature; and 
serum was stored, in aliquots, at $-20^{\circ} \mathrm{C}$ until used.

Sialic acid levels were estimated by Warren's TBH method (Warren, 1959). A calorimetric assay in which sialic acids are oxidized with sodium periodate in concentrated phosphoric acid. The periodate oxidation product is coupled with thiobarbituric acid and the resulting chromophore is extracted into cyclohexanone. The thiobarbituric acid assay is reproducible, sensitive, and considerably more specific than other methods.

Statistical analysis: The results were organized, tabulated and statistically analyzed using Statistical Package for Social Science (SPSS) program version
10, Chicago-IL, USA (Norusis, 1997). Data were presented as $\mathrm{M} \pm \mathrm{SD}$ and percentages when appropriate. Student ttest was used for analysis of two quantitative data. Differences among the two groups were compared by one-way ANOVA followed by post-hoc test. Simple linear correlation (Pearson's correlation for quantitative data and Spearman correlation for qualitative data) was done. ROC (Receiver Operating Characteristic) curve was drawn for detection of reliability of the marker and its best cutoff value.

Specificity and sensitivity were calculated, and AUC (area under the curve) was considered if $>0.60$. $p$ value $\leq 0.05$ was considered significant.

\section{Results}

The main demographic data $(\mathrm{M} \pm \mathrm{SD})$ among the groups are shown in table (1). There was no significant difference between groups as regard age, sex, or smoking.

Table I: Demographic data of groups

\begin{tabular}{|l|l|lc|c|}
\hline Variables & $\begin{array}{l}\text { Malignant Pleural Effusion (n= } \\
30)\end{array}$ & Non-malignant & $\begin{array}{r}\text { Pleural } \\
\text { Effusion } \\
(\mathrm{n}=30)\end{array}$ & $\begin{array}{r}\mathrm{p}- \\
\text { value }\end{array}$ \\
\hline $\begin{array}{l}\text { Age } \\
\text { years })\end{array}$ & $58.4 \pm 13.1$ & $55.3 \pm 11.02$ & 0.7 \\
\hline Sex $(\mathrm{F} / \mathrm{M})$ & $11 / 19$ & $8 / 22$ & 0.8 \\
\hline Smoking & 16 & 19 & 0.8 \\
\hline
\end{tabular}

Table 2: Causes of malignant and nonmalignant pleural effusion among groups

\begin{tabular}{|l|c|c|}
\hline Groups & Causes & Number of cases (\%) \\
\hline Malignant pleural effusion & lung cancer & $25(83.33 \%)$ \\
$(\mathrm{G} \mathrm{I}, \mathrm{n}=30)$ & Mesothelioma & $3(10.00 \%)$ \\
& Esophageal neoplasia & $1(03.33 \%)$ \\
& Mediastinal neoplasia & $1(03.33 \%)$ \\
\hline Nonmalignant pleural effusion & Tuberculosis & $20(66.66 \%)$ \\
$(\mathrm{GII}, \mathrm{n}=30)$ & Pneumonia & $5(16.66 \%)$ \\
& Cardiac failure & $3(10.00 \%)$ \\
& Liver cirrhosis & $1(03.33 \%)$ \\
& Chronic renal failure & $1(03.33 \%)$ \\
\hline
\end{tabular}

Pleural fluid levels of sialic acid were statistically and significantly higher in malignant group compared to nonmalignant one $(76.23 \pm 11.18$ vs. $57.11 \pm$
15.22, $\mathrm{p}<0.05)$. Also, serum levels of sialic acid were statistically and significantly higher in malignant group compared to nonmalignant one $(85.03 \pm$ 
10.04 vs. $70.53 \pm 6.19, \mathrm{p}<0.05)$. Ratio of sialic acid levels in pleural fluid to serum ( $\mathrm{PF} / \mathrm{S}$ ratio) was higher in malignant group $(0.89 \pm 0.068)$ as com- pared to nonmalignant group $(0.81 \pm$ 0.21 ), though difference was not statistically significant (Tab. 3).

Table 3: Sialic acid levels among groups

\begin{tabular}{|l|l|l|l|}
\hline Variable & Malignant Pleural Effusion & Nonmalignant Pleural Effusion & p-value \\
\hline PF sialic acid (mg/dL) & $76.23 \pm 11.18$ & $57.11 \pm 15.22$ & $0.000^{*}$ \\
\hline S sialic acid (mg/dL) & $85.03 \pm 10.04$ & $70.53 \pm 6.19$ & $0.000^{*}$ \\
\hline PF/S sialic acid & $0.89 \pm 0.068$ & $0.81 \pm 0.21$ & 0.059 \\
\hline
\end{tabular}

Values expressed as $\mathrm{M} \pm \mathrm{S} . \mathrm{D}, \mathrm{P}$-value $\leq 0.05^{*}=$ significant, $\mathrm{PF}=$ Pleural fluid, $\mathrm{S}=\mathrm{Serum}, \mathrm{PF} / \mathrm{S}=$ Pleural flu$\mathrm{id} /$ serum ratio

In malignant group, smokers showed a statistically significant higher pleural fluid and serum sialic acid levels as compared to nonsmokers $(81.00 \pm 9.45$ vs. $65.11 \pm 5.55$ and $89.00 \pm 8.95$ vs. $75.78 \pm 5.28$, respectively) $(\mathrm{p}=0.00)$. While $\mathrm{PF} / \mathrm{S}$ sialic acid ratio, in smokers compared to nonsmokers of same group, showed higher but not statistically significant value $(0.91 \pm 0.06$ vs. $0.86 \pm 0.05) \quad(p<0.05)$. In the nonmalignant group (II), no significant change between smokers and nonsmokers was found regarding pleural fluid and serum sialic acid levels and $\mathrm{PF} / \mathrm{S}$ sialic acid ratio (tab. 4).

Table 4: Sialic acid levels among smokers and nonsmokers groups

\begin{tabular}{|l|l|l|l|l|l|l|}
\hline Variables & \multicolumn{2}{l|}{ Malignant Pleural Effusion } & p-value & \multicolumn{2}{l|}{ Nonmalignant Pleural Effusion } & $\mathrm{p}$ value \\
\hline & Smokers & Nonsmokers & & Smokers & Nonsmokers & \\
\hline PF Sialic acid $(\mathrm{mg} / \mathrm{dL})$ & $81.00 \pm 9.45$ & $65.11 \pm 5.55$ & $0.000^{*}$ & $56.36 \pm 15.36$ & $57.86 \pm 15.58$ & 0.793 \\
\hline S Sialic acid $(\mathrm{mg} / \mathrm{dL})$ & $89.00 \pm 8.95$ & $75.78 \pm 5.28$ & $0.000^{*}$ & $70.53 \pm 5.59$ & $70.53 \pm 6.93$ & 1.000 \\
\hline PF/S Sialic acid & $0.91 \pm 0.06$ & $0.86 \pm 0.05$ & 0.061 & $0.809 \pm 0.23$ & $0.824 \pm 0.20$ & 0.857 \\
\hline
\end{tabular}

A statistically significant positive correlation was found between pleural fluid sialic acid and serum sialic acid levels in malignant group (Fig. 1), but no significant correlation could be observed in nonmalignant group.

By using receiver operator characteristic (ROC) analysis, in malignant pleural effusion cut off value of pleural fluid sialic acid was $69.65 \mathrm{mg} / \mathrm{dl}$, sensitivity was $70 \%$, specificity was $96 \%$ and positive predictive value (PPV) was $46.34 \%$ (Fig. 2, Tab. 5). This approved value of pleural fluid sialic acid as a promising marker for diagnosis of malignant pleural effusion.

Table 5: Sensitivity, Specificity, Best Cutoff value and PPV of pleural fluid Sialic acid

\begin{tabular}{|l|l|l|l|l|l|}
\hline AUC & P value & Best Cutoff & Sensitivity & Specificity & PPV \\
\hline 0.884 & $<0.0001 *$ & $69.65(\mathrm{mg} / \mathrm{dL})$ & $70 \%$ & $69 \%$ & $46.34 \%$ \\
\hline
\end{tabular}




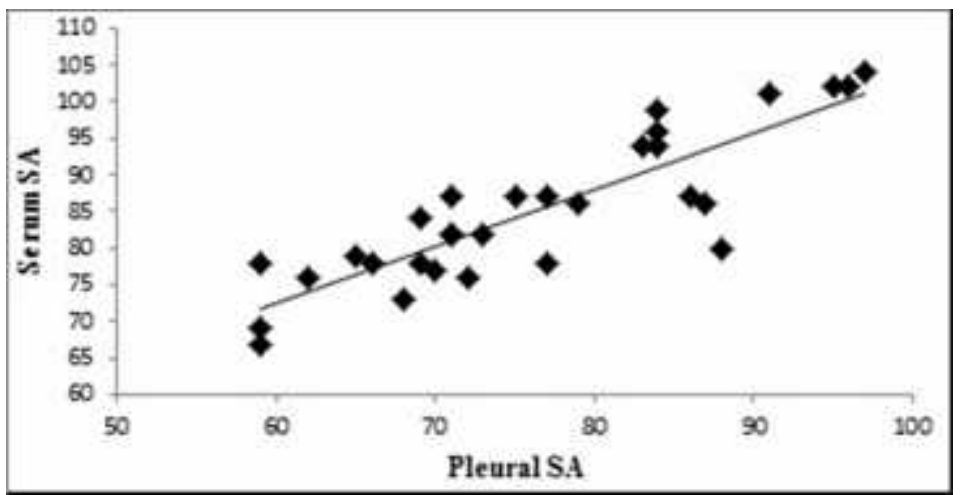

Fig. 1: Correlation between serum sialic acid (SA) and pleural Ssialic acid (SA) among malignant cases

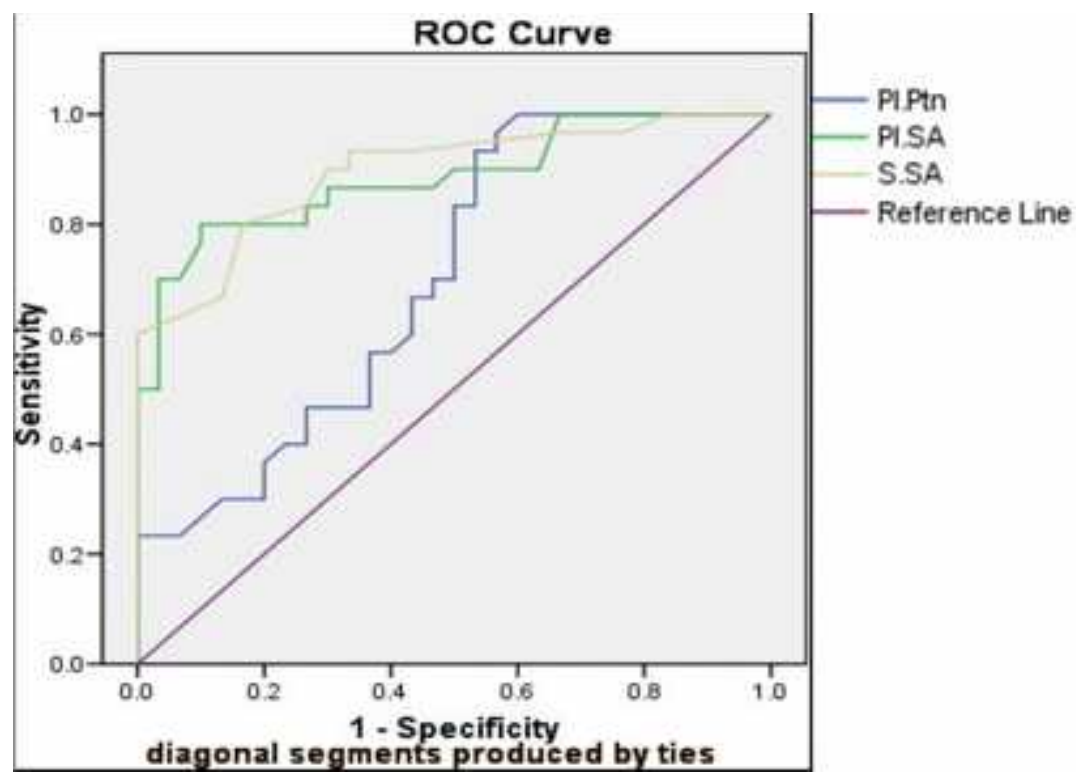

Fig. 2: ROC curve of pleural fluid Sialic acid

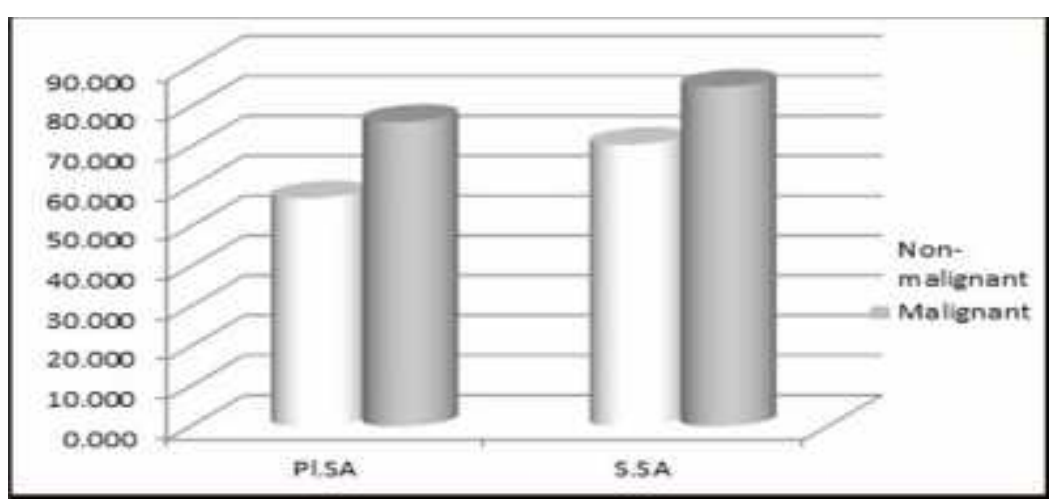




\section{Discussion}

In differential diagnosis of exudative pleural effusions, cytology is the most sensitive method. Since cytology findings are positive in half of such fluids, combined use of reliable tumor marker and cytology is a logical approach.

The determination of tumor markers in pleural effusions has been proposed as an alternative, noninvasive way of establishing a diagnosis of pleural malignancy. However, these measurements in clinical practice remains controversial (David et al, 2005).

Sialic acid and other tumor markers are assuming increasingly important role in clinical oncology, accordingly identification of reliable markers or substances associated with neoplasia is the goal of many researches (Erbil et $a l, 1985)$.

The present study showed a statistically significant increase in sialic acid levels in both pleural fluids and sera of patients with malignant pleural effusion compared to nonmalignant effusion. Also, $\mathrm{PF} / \mathrm{S}$ sialic acid ratio was higher in malignant group compared to nonmalignant but with no significant difference. These results agreed with Krolikowski et al. (1976) who showed a significant difference in the levels of sialic acid in patients with lung cancer compared to normal controls. Hence they reported sialic acid to be of value as a biologic marker in lung cancer. Seber et al. (1989) also showed that sialic acid levels in bronchalveolar lavage fluid were highly correlated with the diagnosis of bronchial cancer. Kakari et al. (1991) determined total sialic acid (TSA) and lipid-bound sialic acid
(LSA) concentrations in comparison to CEA levels in 152 untreated patients with primary lung cancer and 107 benign pulmonary diseases. They concluded that although TSA and LSA were highly sensitive markers of lung cancer, their specificity was low in this setting. Imecik and Ozer (1992) measured sialic acid levels in the pleural effusions from patients with malignant and benign lung pathologies. Finding higher sialic acid concentrations in malignant pleural effusions than in those of benign origin, they concluded that the sialic acid had diagnostic value. Patel et al. (1995) showed that carcinoembriyonic antigen (CEA) and phosphohexose isomerase (PHI) levels along with total sialic acid/total protein ratios (TSA/TP) in 192 untreated lung cancer patients and in 80 age- and sexmatched control cases were compared. PHI and TSA/TP were found to be as an important tumor marker in patients with lung cancer.

Also, the present data agreed with Imecik and Ozer (1992) who found 33.33\% of cases of malignant pleural effusions had raised pleural fluid sialic acid as compared to serum levels which were in the normal range. This could be either due to high production and gradual absorption of sialic acid in the diseased area or elevation of both serum and pleural fluid levels, but sialic acid disappears more slowly from pleural fluid. Also, Bektemur et al. (2003) reported high serum sialic acid levels in malignant pleural effusions though difference was not statistically significant. They also observed high pleural fluid 
sialic acid levels and PF/S sialic acid ratio.

On the contrary, Isitmangil et al. (2001) reported that sialic acid levels in bronchoalveolar lavage fluid and serum did not show any statistically significant difference between subjects with malignant and the non-malignant lung diseases $(p>0.05)$. Sialic acid levels were also unrelated to the stage and localization of the tumor $(\mathrm{p}>$ 0.05 ). They concluded that sialic acid levels did not appear to be a good marker for discriminating malignant from nonmalignant diseases of the lung.

The current study showed a significant higher pleural fluid and serum sialic acid levels in smokers as compared to nonsmokers of the malignant group $(\mathrm{p}<0.001)$, also, the $\mathrm{PF} / \mathrm{S}$ sialic acid ratio, in smokers compared to nonsmokers of the same group, showed higher but not statistically significant value. While no significant changes were detected for the same parameters in the nonmalignant group between smokers and nonsmokers.

In smokers serum sialic acid increased since smoking induces tissue inflammation in addition of being known as carcinogenic (Crook et al, 2000; Kurtul, et al, 2004).

By using ROC analysis, the present study found that in malignant pleural effusion the cut off value of pleural fluid sialic acid was $69.65 \mathrm{mg} / \mathrm{dL}$, sensitivity was $70 \%$, and specificity was 96\%. This agreed with Bektemur et al. (2003) who reported that in differential diagnosis of malignant pleural effusions, sensitivity and specificity of pleural fluid sialic acid were $61 \%$, and $91 \%$, respectively.

Also, Bansal et al. (2010) while taking the cut off value of $70 \mathrm{mg} / \mathrm{dL}$ for pleural fluid sialic acid in malignant pleural effusions, they found that the sensitivity was $63.33 \%$, specificity $60 \%$, and they concluded that determination of sialic acid levels in pleural fluid has diagnostic value as a cheap, simple and reliable marker for malignant pleural effusion.

\section{Conclusion}

The outcome findings suggests that determination of sialic acid levels in pleural fluid has diagnostic value as being cheap, simple and reliable marker for malignant pleural effusion and combined use of sialic acid with cytology is highly recommended for further research evaluation

\section{References}

Bansal, A, Tandon S, Kharb, S, 2010: Diagnostic value of sialic acid in pleural effusion. Chin. J. Lung Cancer; 13: 349-351.

Bektemur, G, Ozer, F, Kanat, F, et $a l$, 2003: Diagnostic efficiency of serum lipid bound sialic acid level in malignant pleural effusions. Tuberk. Toraks. 51:265-70.

Crook, M, Scott, D, Stapleton, J, et al, 2000: Circulating concentrations of $\mathrm{C}$-reactive protein and total sialic acid in tobacco smokers remain unchanged following one (1) year of validated smoking cessation. Eur. J. Clin. Invest. 30:861-5.

David, S, Boris, Z, Ariella, S, et al, 2005: Diagnostic value of CYFRA 211, CEA, CA 199, CA 15-3, and CA 
125 assays in pleural effusions. Oncologist 10:501-7.

Emmet, E, Paul, B, 2011: Diagnosis of Pleural Effusion: A Systematic Approach. Am. J. Crit. Care 20:119-28.

Erbil, M, Jones, J, Klee, G, 1985: Use and limitations of serum total and lipidbound sialic acid concentrations as markers for colorectal cancers. Cancer 55:404-9.

Imecik, O, Ozer, F, 1992: Diagnostic value of sialic acid in malignant pleural effusions. Chest 102:1819-22.

Isitmangil, T, Isitmangil, G, Budak, Y, et al, 2001: Comparison of serum and broncho-alveolar lavage fluid sialic acid levels between malignant and benign lung diseases. BMC Pulmonary Med.4:29-33.

Kakari, S, Stringou, E, Toumbis, M, et al, 1991: Five tumor markers in lung cancer: significance of total and lipidbound sialic acid. Anticancer Res. 11: 2107-10.

Krolikowski, F, Reuter, K, Waales, T, et al, 1976: Serum sialic acid levels in lung cancer patients. Pharmacol. 14: 47-51.

Kurtul, N, Cil, M, Bakan E, 2004: The effects of alcohol and smoking on serum, saliva, and urine sialic acid levels. Saudi Med. J. 25:1839-44.

Light, R, 1995: Malignant pleural effusion. In: Light RW, ed. Pleural Diseases, $3^{\text {rd }}$ Edition. Baltimore: Williams and Wilkins.

Marel, M, Stastny, B, Melinova, L, et al, 1995: Diagnosis of pleural effusions: Experience with clinical studies. Chest 107:1598-1603.
Nicola, B, Prasad, S, 2002: Sialic acid changes in Dalton's lymphoma-bearing mice after cyclophosphamide and cisplatin treatment. Brazilian J. Med. Biol. Res. 35:549-53.

Norusis, J, 1997: SPSS 7.5 guide to data analysis, Simon and Schuster Co/, Upper Saddle River. New Jersey.

Patel, P, Raval, G, Rawal, R, et al, 1995: Comparison between serum levels of carcinoembryonic antigen, sialic acid and phosphohexose isomerase in lung cancer. Neoplasma 42:271-4.

Seber, O, Aydilek, R, Erbil, K, et al, 1989: Diagnostic value of lipid bound sialic acid levels in serum and bronchial lavage fluid in bronchial cancer. Asian Med. J. 32:109-15.

Selva, C, Kalaivani, R, 2011: Study of Adenosine Deaminase and Serum Protein Bound Sialic Acid Levels in Alcoholic Liver Disease. Int. J. Biol. Med. Res. 2:754-6.

Silver H., Karim K., Archibald E. et al. (1979): Serum sialic acid and sialyltransferase as monitors of tumor burden in malignant melanoma patients. Cancer Res.1979; 39:5036-5042.

Villena, V, Lopez-Encuentra, A., Echave-Sustaeta, J, et al, 1996: Diagnostic value of CA 72-4, carcinoembryonic antigen, CA 15-3, and CA 19-9 assay in pleural effusion: Study of 207 patients. Cancer 78:736-40.

Vinaya, S, Jyotsna, M, 2012: Pleural effusion: diagnosis, treatment, and management. Open Access Emerg. Med. 4:31-52.

Warren, L, 1959: The thiobarbituric acid assay of sialic acid. J. Biol. Chemist. 234: 1971-5. 